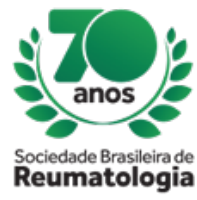

\title{
CHIKUNGUNYA FEVER IN PATIENTS UNDER BIOLOGICAL THERAPY: POSSIBLE PROTECTIVE EFFECT?
}

Claudia Marques (Rheumatology, Clinical Hospital of Pernambuco Federal University, Recife, PE, Brasil), Janaina Monteiro (Medicine undergraduate student, Pernambuco Federal University, Recife, PE, Brasil), Marina Brito (Medicine undergraduate student, Pernambuco Federal University, Recife, PE, Brasil), Fabiana Lopes (Medicine undergraduate student, Pernambuco Federal University, Recife, PE, Brasil), Anton Lima (Medicine undergraduate student, Pernambuco Federal University, Recife, PE, Brasil), Gabriel Marques (Medicine undergraduate student, Universidade Católica de Pernambuco (UNICAP), Recife, PE, Brasil), Maria Luisa Valadares (Medicine undergraduate student, Faculdade de Medicina de Olinda (FMO), Recife, PE, Brasil), Raquel Carrera (Centro de Pesquisas Aggeu Magalhães - FIOCRUZ/PE, Recife, PE, Brasil), Rafael França (Centro de Pesquisas Aggeu Magalhães - FIOCRUZ/PE, Recife, PE, Brasil), Carlo Brito (Clinical Hospital of Pernambuco Federal University, Recife, PE, Brasil), Angela Duarte (Rheumatology, Clinical Hospital of Pernambuco Federal University, Recife, PE, Brasil)

\section{BACKGROUND}

Little is known about the effect of the use of immunobiological medications in patients with rheumatic diseases that are infected by the Chikungunya virus (CHIKV). Despite initial concerns, the clinical practice and a few case reports demonstrated no major complications with its use. Besides that, some patients under immunobiological therapy reported: "everyone in my house got sick (with Chikungunya), except me", suggesting a possible protective effect of biological therapy. The aim of this study was to evaluate the frequency of subclinical CHIKV infection in patients using immunobiological agents and compared to controls living in the same house, as well the possible protective effect of these medications on Chikungunya fever clinical course.

\section{MATERIALS AND METHODS}

Cross-sectional, observational, analytical study carried out from January to June 2018. Patients ?18 years old with diagnosis of rheumatologic disease treated with biological therapy were included; the control group was composed of people who lived in the same house of the patient. Data were collected about symptoms suggestive of acute CHIKV infection in the past, use of medications and then blood was collected to perform the specific serology for CHIKV IgG.

\section{RESULTS}

A total of 168 patients were evaluated, most of them with rheumatoid arthritis (94; 56.0\%); the most frequent class of immunobiological used was anti-TNF (47.0\%). Of the 168 cases, 42 presented CHIKV positive IgG serology (25.0\%) and 9 of them had no symptoms (5.0\%); among 56 controls, $27.0 \%$ presented positive serology, and only 1 of them did not present symptoms (1.0\%) ( $p=0.2629)$. No significant difference was observed between Chikungunya fever symptoms in the comparing patients using immunobiological therapy and controls. Regarding the duration of Chikungunya fever symptoms, in $72 \%$ of the individuals treated with biological medication the CHIK symptoms improved completely before 3 months while in the control group this occurred in $36.0 \%(p=0.01)$. When stratified by biological class, $95.0 \%$ of those who used anti-TNF improved before 3 months; in those who used other classes, this percentage was $33.0 \%(p=0.001)$.

\section{CONCLUSION}

The use of immunobiological therapy had associated with increased the number of cases of subclinical infection by CHIKV, although without statistical significance. In addition, patients taking anti-TNF showed a shorter $\mathrm{CHIK}$ evolution time when compared to controls and other immunobiological. These findings 
suggest that there may be a possible protective role for this class of drugs in the presence of CHIKV infection. 